\title{
Estimation of stress-dependent anisotropy from P-wave measurements on a spherical sample
}

\author{
Dariush Nadri ${ }^{1}$, Andrej Bóna ${ }^{2}$, Miroslav Brajanovski ${ }^{2}$, and Tomáš Lokajíček ${ }^{3}$
}

\begin{abstract}
Our aim is to understand the stress-dependent seismic anisotropy of the overburden shale in an oil field in the North West Shelf of Western Australia. We analyze data from measurements of ultrasonic P-wave velocities in 132 directions for confining pressures of $0.1-400 \mathrm{MPa}$ on a spherical shale sample. First, we find the orientation of the symmetry axis, assuming that the sample is transversely isotropic, and then transform the ray velocities to the symmetry axis coordinates. We use two parameterizations of the phase velocity; one, in terms of the Thomsen anisotropy parameters $\alpha, \beta, \varepsilon, \delta$ as the main approach, and the other in terms of $\alpha, \beta, \eta, \delta$. We invert the ray velocities to estimate the anisotropy parameters $\alpha, \varepsilon, \delta$, and $\eta$ using a very fast simulated reannealing algorithm. Both approaches result in the same estimation for the anisotropy parameters but with different uncertainties. The main approach
\end{abstract}

is robust but produces higher uncertainties, in particular for $\eta$, whereas the alternative approach is unstable but gives lower uncertainties. These approaches are used to find the anisotropy parameters for the different confining pressures. The dependency of P-wave velocity, $\alpha$, on pressure has exponential and linear components, which can be contributed to the compliant and stiff porosities. The exponential dependence at lower pressures up to $100 \mathrm{MPa}$ corresponds to the closure of compliant pores and microcracks, whereas the linear dependence at higher pressures corresponds to contraction of the stiff pores. The anisotropy parameters $\varepsilon$ and $\delta$ are quite large at lower pressures but decrease exponentially with pressure. For lower pressures up to $10 \mathrm{MPa}, \delta$ always is larger than $\varepsilon$; this trend is reversed for higher pressures. Despite the hydrostatic pressure, the symmetry axis orientation changes noticeably, in particular at lower pressures.

\section{INTRODUCTION}

The main motivation for this study is to understand the stressdependent seismic anisotropy of the overburden shale in an oil field in the North West Shelf of Western Australia. As a result of the depositional pattern of clay minerals, flat-lying shale formations often show transverse isotropy with a vertical axis of symmetry. To characterize such seismic anisotropy, we build upon our work (Bóna et al., 2010) of Thomsen anisotropy parameter estimation using quasi-P-wave ultrasonic traveltimes to study the anisotropy at different confining pressures from a spherical shale sample from just above a sand reservoir.

The idea of studying the velocity anisotropy and estimating the elasticity tensor from the traveltimes on spherical samples has been addressed by different authors. Perhaps the earliest major contributions in this field could be attributed to Pros and
Babuška (1967, 1968), Thill et al. (1969, 1973), Vickers and Thill (1969), and Pros and Podroužková (1974). They designed new techniques and apparatus to measure P-wave velocities on spherical rock samples. They also computed the P-wave velocity anisotropy from the maximum and minimum of the velocity, after finding the symmetry axis in igneous and metamorphic rocks. Jones and Wang (1981) measured the P-waves at normal, parallel, and $45^{\circ}$ to the bedding plane on two Cretaceous shale samples from the Williston Basin at the confining pressures ranging from 1 bar to $4 \mathrm{kbar}$ in dry and saturated conditions. They also measured shear waves in directions normal and parallel to the bedding plane (with polarization lying in the symmetry plane) at the same pressure and saturation conditions as the P-waves. Assuming that the shales are transversely isotropic, they computed five elasticity parameters at the confining pressure of 1 bar.

Manuscript received by the Editor 30 July 2010; revised manuscript received 12 November 2010; published online 23 May 2011.

${ }_{2}^{1}$ CSIRO Earth Science and Resource Engineering, Perth, Australia. E-mail dariush.nadri@csiro.au.

${ }^{2}$ Curtin University, Department of Exploration Geophysics, Perth, Australia. E-mail: a.bona@curtin.edu.au; brajanom@hotmail.com.

${ }^{3}$ Czech Academy of Sciences, Prague, Czech Republic. E-mail tl@gli.cas.cz.

(C) 2011 Society of Exploration Geophysicists. All rights reserved. 
Jech (1991) suggested a nonlinear iterative least-squares optimization procedure to solve the Christoffel equation (see, e.g., Cervený, 1972) for the 21 elasticity parameters from the quasi$\mathrm{P}$-wave traveltimes measured on spherical rock samples. He used the same measurement configuration and equipment as that proposed and designed by Pros and Babuška (1967) and Pros and Podroužková (1974) for high-pressure measurements.

Methodology to estimate the 21 elasticity parameters suggested by Jech (1991) by using the well-distributed quasi-Pwaves may fail for more symmetric anisotropy classes. For example, for a transversely isotropic material it is impossible to estimate $C_{66}$ from only P-wave velocities. Arts et al. (1991, 1996) and Arts (1993) used a method based on the measurements of ultrasonic velocities in a large number of directions on spherical rock samples. Before a sample was made into a sphere, they performed two shear-wave measurements on a cubic sample of the same rock in three mutually perpendicular symmetry directions of the cube using the experimental setup of Lucet (1989) and Lucet and Zinszner (1992). This gives six additional shear-wave measurements.

The $\mathrm{P}$ - and S-wave data are sufficiently redundant to be inverted in terms of the complete set of the 21 elastic coefficients. Vestrum (1994); Vestrum et al. (1996) demonstrated that by using a large number of high-quality P- and S-wave velocity measurements, he could successfully invert for the complete set of 21 elasticity parameters that best fit the velocity measurements in the least-squares sense. His method makes no prior assumptions about the symmetry or orientation of the rock.

Dellinger et al. (1998) modified the algorithm of Vestrum to stabilize the inversion. Rasolofosaon and Zinszner (2002) developed a technique for measuring the complete set of the 21 elastic coefficients, a technique allowing the identification and orientation of the symmetry elements (planes, axes), and a technique for approximating the derived elastic tensor by a tensor of higher symmetry with the quantification of the error induced by such an approximation.

Experimental studies on shale to estimate elasticity/Thomsen anisotropy parameters at different pressures are not widely addressed in the geophysical literature.

Among these, Hornby (1998) measures one P-wave and two $\mathrm{S}$-wave velocities for three cylindrical samples from the same shale cut at normal, parallel, and $45^{\circ} / 30^{\circ}$ to the symmetry axis under the confining pressure from 5 to $80 \mathrm{MPa}$ to estimate the elasticity and Thomsen anisotropy parameters. This data set was used by Prioul et al. (2004) to propose a nonlinear rock physics model to predict stress-dependent elasticity parameters for transverse isotropic shale under isotropic and anisotropic stress fields. This data set was used by Ciz and Shapiro (2009) to address the stress-induced anisotropy for transversely isotropic elasticity using an anisotropic extension of the porosity deformation formalism (Shapiro, 2003). Wang (2002a, 2002b) proposed a single core plug measurement of P-wave at normal, parallel, and $45^{\circ}$ to the bedding plane and also two shear waves along the bedding plane to measure the elasticity parameters for a transversely isotropic rock sample. He has computed the elasticity parameters for a large number of samples from shale and reservoir rocks at different fluid saturations and effective pressures.

Sarkar et al. (2003) studied Thomsen anisotropy parameter variations under nonhydrostatic stress conditions on Berea sandstone. The sample was subjected to uniaxial stress fields up to
$10 \mathrm{MPa}$, causing orthorhombic seismic anisotropy. They provide a theoretical model that decouples the stress-induced anisotropy from the background transverse isotropy with vertical symmetry axis (VTI) under different uniaxial stress fields. They also have estimated the Thomsen anisotropy parameters $\varepsilon, \delta$, and $\gamma$ at the different symmetry planes, and have decoupled the portions of these parameters from the VTI background and the stress-related component. The stress-related component of Thomsen anisotropy parameters in the major symmetry plane has been shown to be proportional to the magnitude of the stress field.

Dewhurst and Siggins (2006) have conducted similar experimental studies on shale core samples under uniaxial and isotropic stress fields. Assuming the shales are transversely isotropic, they measured $\mathrm{P}$-waves in directions parallel, normal, and $45^{\circ}$ to the bedding plane, and two shear waves parallel to the bedding plane, at different net stresses to compute the transversely isotropic elasticity tensor and the Thomsen anisotropy parameters. Despite the simplicity of these types of ultrasonic measurements they suffer from the lack of robustness; Bóna et al. (2010) show how a limited number of ultrasonic measurements can propagate errors in estimation of elasticity parameter $C_{13}$ and Thomsen anisotropy parameters, in particular $\delta$.

Herein, we present a method of inversion of the P-wave traveltimes for the Thomsen anisotropy parameters that differs from the above approaches in two major ways. First, we use the numerical derivatives of the measured traveltimes with respect to the ray angles to express the phase angles using only measured quantities. Secondly, unlike in our previous approach (Bóna et al., 2010), where we estimate the Thomsen anisotropy parameters only at the reservoir pressure, we estimate the Thomsen anisotropy parameters at every pressure. We use the very fast simulated reannealing as a global optimization approach to overcome the nonuniqueness and compare it to local optimization algorithms such as nonlinear conjugate gradient and quasiNewton methods. Both of these differences result in a more robust estimate of Thomsen anisotropy parameters than do alternative approaches.

\section{ULTRASONIC MEASUREMENT CONFIGURATION}

A core plug from overburden at the depth of $2076 \mathrm{~m}$ was chosen for our anisotropy studies. This sample is a fairly homogenous argillaceous siltstone aged late Jurassic to early Cretaceous, and is deposited in a lower delta plain to open marine environment in Exmouth Basin, North West Shelf of Western Australia. We often refer to this sample as shale. The neutron porosity from the well $\log$ corresponding to the depth of this sample is $38 \%$ and the gamma ray is 135 API. Density is $2.45 \mathrm{~g} / \mathrm{cc}$ from the $\log$ and laboratory measurements.

Before making a spherical sample, the core plug was vacuumed, but was not dried completely and some pores might be saturated partially with brine. We assume that increasing the confining pressure essentially leaves the pore pressure unchanged. To prepare a spherical sample, the core plug is polished in different directions to form a sphere with $50 \pm 0.01$ $\mathrm{mm}$ diameter.

Figure 1 shows two computer tomography (CT) images from the sample at ambient pressure. These images were taken after the experiment. The light and dark colors correspond to higher and lower density minerals, respectively. The resolution of the 
images is not good enough to see the minerals individually; however the lamination is visible.

The spherical sample was covered by a thin epoxy resin film (thickness $0.05 \mathrm{~mm}$ ) to protect the pore space from the pressure medium and was placed in an oil-filled pressure chamber at room temperature to measure the ultrasonic $\mathrm{P}$-wave velocities in a broad range of confining pressures, from ambient pressure to $400 \mathrm{MPa}$.

Figure 2 shows a schematic of the measurement locations on the spherical sample. Two similar piezoceramic transducers were placed at the opposite sides of the spherical sample. A high-voltage pulse excited one of the transducer-generated ultrasonic waves propagating through the sample and recorded at the other transducer. Signals were measured over the spherical sample at every $15^{\circ}$ in azimuthal and polar directions. This acquisition pattern produces 132 records of P-waveforms sampled at 10 ns. From these seismograms we picked the first arrivals by means of a high-order statistics approach (Lokajíček and Klíma, 2006) and then found the ray velocities. The measurements on the spherical sample were performed at the Institute of Geology, Czech Academy of Sciences, Prague. A more comprehensive description of the experimental setup is given by Pros et al. (1998).

\section{ESTIMATION OF SYMMETRY AXIS}

Following Bóna et al. (2010), we choose to invert the measured velocities for the transversely isotopic tensor in two steps. In the first step we find the symmetry axis, and in the second step we find the Thomsen anisotropy parameters. These steps could be combined into one step; however, we prefer to keep them separate to reduce the complexity of the inversion.

First, we need to establish whether we measure phase or ray (also called group) velocities. This can be determined by comparing the diameter of the transducer with the diameter of the sample. In our case, the active area of the piezoelectric element is $3 \mathrm{~mm}$ in diameter, which is $1 / 17$ of the diameter of the sample, and the natural frequency of the transmitter is $2.5 \mathrm{MHz}$. Following Vestrum (1994), we conclude that the measured velocities are the ray velocities.

To estimate the symmetry axis of a transversely isotropic medium, we use the fact that the wavefronts must be at least as symmetric as the material itself (Bóna et al., 2007). To find the symmetry axis, assuming this spherical shale sample has the transverse isotropy symmetry, we form an objective function
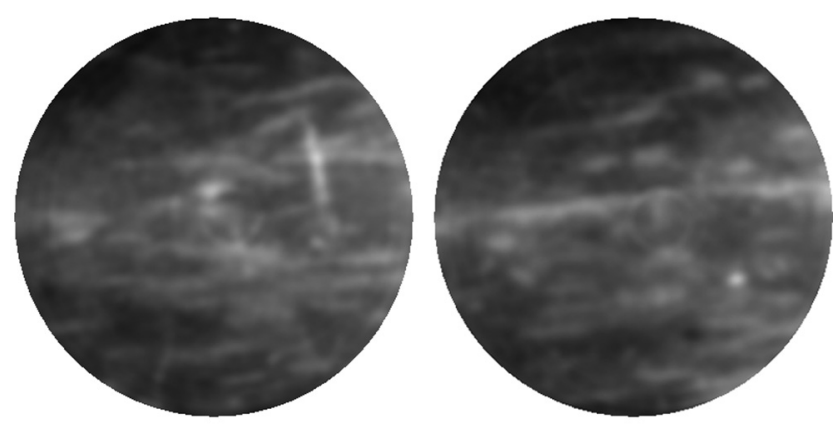

Figure 1. CT images from two slices of the spherical shale sample. The diameter of the slices is $47.2 \mathrm{~mm}$.

$$
S\left(\psi_{0}, \omega_{0}\right)=\sum_{\psi=0}^{2 \pi} \sum_{\omega=0}^{\pi}\left(\frac{\partial \psi}{\partial \varphi} \frac{\partial V}{\partial \psi}+\frac{\partial \omega}{\partial \varphi} \frac{\partial V}{\partial \omega}\right)^{2},
$$

where $V$ is the ray velocity, $\psi_{0}$ and $\omega_{0}$ are the assumed coordinates of the symmetry axis, $\psi, \omega$ are the measurement coordinates, and $\zeta, \varphi$ are the symmetry coordinates, as shown in Figure 3. The symmetry axis is found by minimizing this function. To find the minimum of $S$, we change the orientation of the azimuth axis. This requires transforming the data from the measurement coordinate system to the symmetry coordinate system for a given rotation (Figure 3). The objective function $S$ is $2 \mathrm{D}$, and direct searching of the model space was used to find the solution.

Table 1 shows the symmetry coordinates from the measurements at different pressures. Figures 4 and 5 show small but systematic changes in the direction of the symmetry axis with pressure. The change is rapid up to $40 \mathrm{MPa}$, at which point the change reverses and slows at the higher pressures. Although the pressure field is isotropic, it changes the symmetry axis direction. The scattered

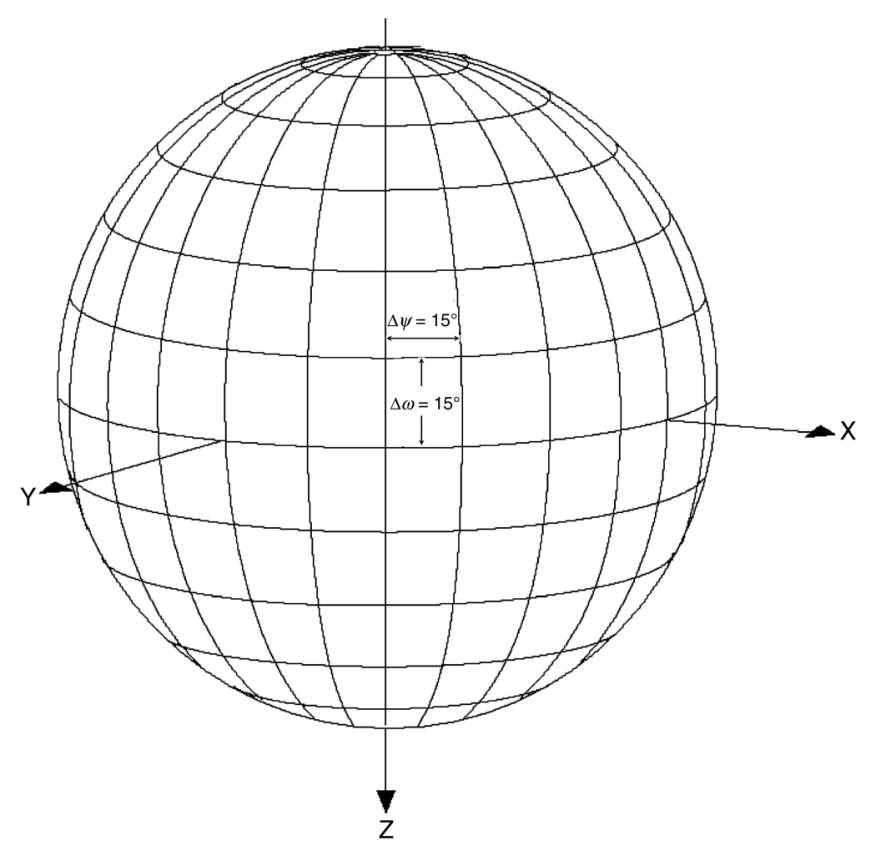

Figure 2. A schematic of measurement locations on the sphere. a)

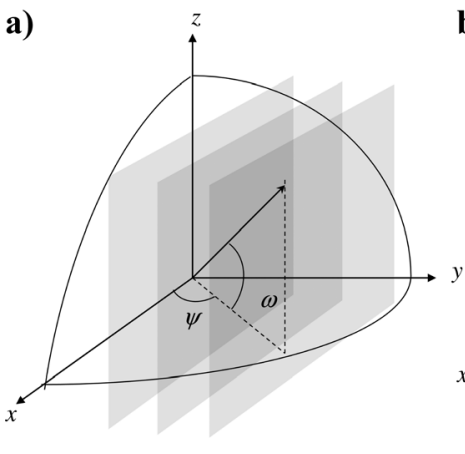

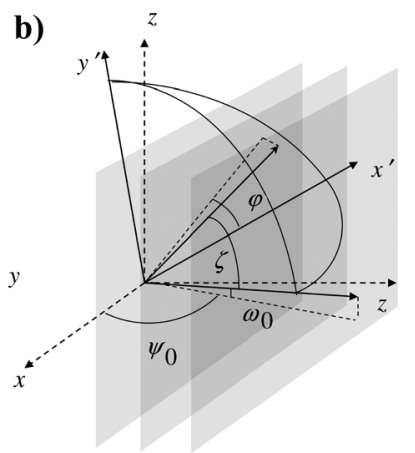

Figure 3. (a) Measurement and (b) symmetry axis coordinate systems after rotation $\left(\psi_{0}, \omega_{0}\right)$. Vertical planes are symmetry planes. The symmetry axis is denoted by $z^{\prime}$. 
points at $70 \mathrm{MPa}$ for $\psi_{0}$ and $300 \mathrm{MPa}$ for $\omega_{0}$ could be explained by propagation of measurement errors through the numerical derivatives in expression 1 .

\section{ESTIMATION AND UNCERTAINTY ANALYSIS OF THOMSEN ANISOTROPY PARAMETERS}

In the preceding section we found the best approximation of the symmetry axis of the material under assumption of transverse isotropy. In this section we focus on inversion of the Thomsen anisotropy parameters $\alpha, \varepsilon$, and $\delta$ from the measured data. To this end, we express the measured ray velocity explicitly in terms of the ray angle, which will allow us to construct an objective function for the inversion.

Phase velocity $v$ for a quasi-P-wave as a function of elasticity parameters can be found by solving the Christoffel's equations for a transverse isotropy medium. We use the same notation (Thomsen, 1986),

$$
\begin{gathered}
v^{2}=\alpha_{0}^{2}\left(1+\varepsilon \sin ^{2} \theta+\Delta\right) \\
\Delta=\left(\frac{1-\beta_{0}^{2} / \alpha_{0}^{2}}{2}\right)\left[\left\{1+\frac{4(2 \delta-\varepsilon) \sin ^{2} \theta \cos ^{2} \theta}{1-\beta_{0}^{2} / \alpha_{0}^{2}}\right.\right. \\
\left.\left.+\frac{4\left(1-\beta_{0}^{2} / \alpha_{0}^{2}+\varepsilon\right) \varepsilon \sin ^{4} \theta}{\left(1-\beta_{0}^{2} / \alpha_{0}^{2}\right)^{2}}\right\}^{\frac{1}{2}}-1\right]
\end{gathered}
$$

where $\alpha_{0}, \beta_{0}, \delta, \varepsilon$ are the Thomsen anisotropy parameters, and $\theta$ is the phase angle (wavefront normal angle) measured from the symmetry axis.

Because of the relatively small size of the transducers, the measured velocity is the ray velocity. The magnitude of the ray velocity is related to the phase velocity (Berryman, 1979)

Table 1. Symmetry axis coordinate $\left(\psi_{0}, \omega_{0}\right)$ estimated from the $P$-wave ray velocities at different confining pressures. ${ }^{3}$

\begin{tabular}{lcc}
$P_{c}(\mathrm{MPa})$ & $\psi_{0}^{\circ}$ & $\omega_{0}^{\circ}$ \\
\hline 0.1 & 82.2 & -3.1 \\
1 & 80.7 & -3.6 \\
2 & 81.2 & -2.9 \\
4 & 81.5 & -2.9 \\
7 & 81.6 & -2.5 \\
10 & 83.6 & -2.5 \\
15 & 83.2 & -2.3 \\
20 & 84.5 & -2.3 \\
40 & 84.8 & -2.2 \\
70 & 83.6 & -2.2 \\
100 & 84.8 & -2.8 \\
200 & 83.8 & -4.9 \\
300 & 83.4 & -3.0 \\
400 & 82.2 & -6.4 \\
\hline
\end{tabular}

${ }^{3}$ The angles are measured in the measurement coordinate system, which was established visually, based upon the lamination of the shale.

$$
V^{2}=v^{2}+(\partial v / \partial \theta)^{2}
$$

where $V$ is the ray velocity and $\partial v / \partial \theta$ can be computed from equation 2. To express phase angle as a function of ray angle and ray velocity, we follow the same approach as Ursin and Hokstad (2003),

$$
\cos ^{2} \theta=\left(\cos \zeta+\sin \zeta \frac{d \ln V}{d \zeta}\right)^{2} /\left(1+\left(\frac{d \ln V}{d \zeta}\right)^{2}\right)
$$

where $\zeta$ is the ray angle. The derivative $d \ln V / d \zeta$ can be found numerically from the measured data, e.g., by using a four-term finite-difference operator. Substituting expression 4 into expression 2 and the result into expression 3, we obtain the ray velocity expressed as a function only of the measured quantities and

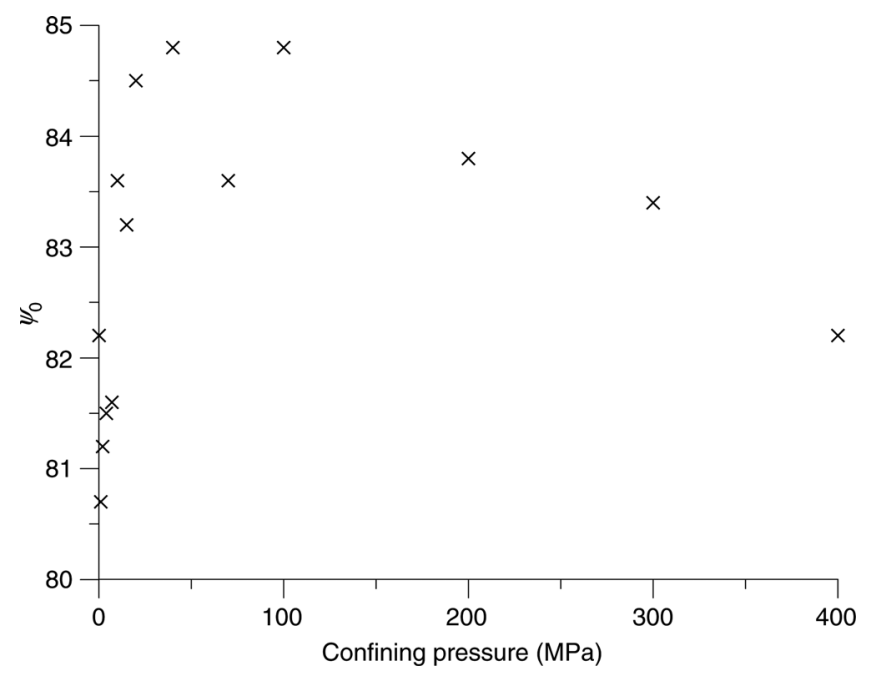

Figure 4. Azimuth, $\psi_{0}$, of the symmetry axis coordinate at different confining pressures.

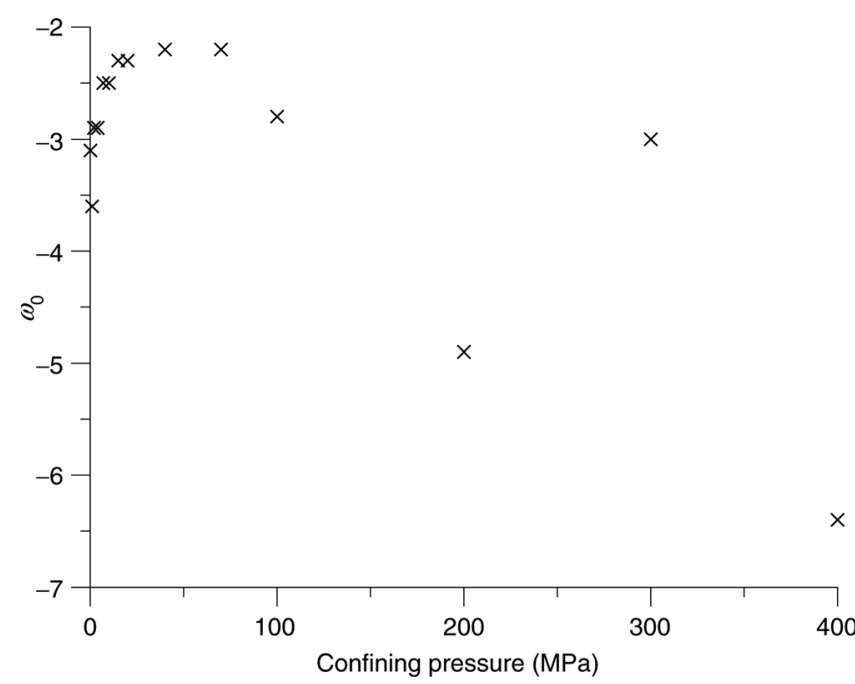

Figure 5. Polar angle (dip), $\omega_{0}$, of the symmetry axis coordinate at different confining pressures. 
the unknown Thomsen anisotropy parameters. This way of expressing the ray velocity is one of the main advantages of the present method: We can invert directly for Thomsen anisotropy parameters without the need to calculate the phase angles at each step of the inversion process. As a result of the lack of enough measurements along the polar direction (angle from the symmetry axis) in each azimuth, we use linear triangular interpolation for smaller $\Delta \theta$.

We estimate the Thomsen anisotropy parameters $\alpha, \varepsilon$, and $\delta$ from the measured P-wave ray velocities. We estimate the three parameters by minimizing a quadratic objective function of residual errors given by Tarantola (2005),

$$
f=\frac{1}{2}\left(\boldsymbol{V}-\boldsymbol{V}^{\prime}\right)\left[\mathbf{C}_{\mathbf{D}}\right]^{-1}\left(\boldsymbol{V}-\boldsymbol{V}^{\prime}\right)^{T},
$$

where $\boldsymbol{V}$ and $\boldsymbol{V}^{\prime}$ are the vectors of $N$ measured and computed ray velocities in symmetry axis coordinates over the entire azimuth and polar angles, respectively. The symbol $\mathbf{C}_{\mathbf{D}}$ is the $N \times N$ covariance matrix of the data. We assume that there is no correlation between the errors in data, so the off-diagonal elements of the covariance matrix are zero. The diagonal elements, or the variances $\sigma^{2}$, could be the errors in picking the traveltimes, or here, the error in measuring the ray velocities. The uncertainty $\sigma$ in ray velocities is kept at $0.05 \mathrm{~km} / \mathrm{s}$ for all the ray velocities, which results from the error in laboratory measurement and traveltime picking.

\section{Sensitivity analysis}

To determine the sensitivity of the objective function (5) to the model parameters, we explore the model space with different model parameters. The sensitivity analysis shows that shear-wave velocity $\beta$, as expected (e.g., Tsvankin, 2005), has very little effect on the objective function, and thus the estimate of $\beta$ is not reliable in the presence of noise. Figure 6 shows the model space for a given $\beta=1.5 \mathrm{Km} / \mathrm{s}$. The iso-surface has been set at the vicinity of minimum to show the behavior of the objective function. The parameters $\alpha, \varepsilon$, and $\delta$ have different uncertainty, but all converge to their solution. The estimation of Thomsen anisotropy parameters has the advantage over the estimation of elasticity parameters and then mapping them to Thomsen anisotropy parameters, since correlation between $C_{13}$ and $C_{44}$ requires an accurate estimate of $C_{44}$ from other sources, or having shear-wave measurements as well (Bóna et al., 2010).

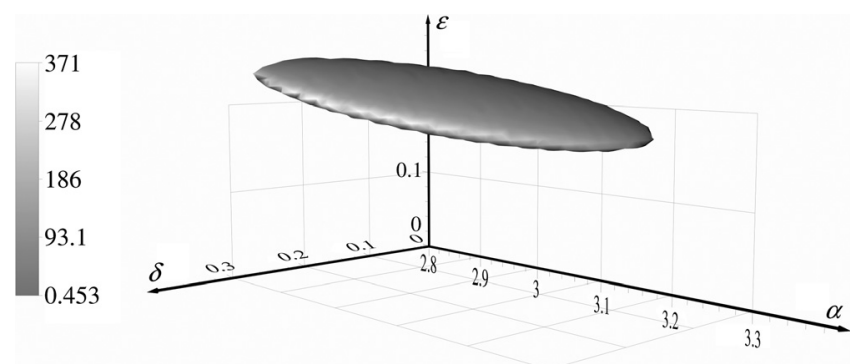

Figure 6. A closed iso-surface (for the objective function value 1.55) of the model space in the vicinity of the solution where the value of the Thomsen anisotropy parameter $\beta$ is kept at $1.5 \mathrm{~km} / \mathrm{s}$. Though the uncertainty varies for different parameters, they can be estimated very well. This solution is practically independent of $\beta$.

\section{Local and global minimization}

To minimize the objective function given by expression 5 , we implemented and compared three algorithms; very fast simulated reannealing (VFSR) algorithm (Ingber, 1989) as a global optimization method, and two local gradient-based search algorithms (Nocedal and Wright, 1999). VFSR and the gradient-optimization methods require derivatives of the objective function with respect to model parameters, which are computed analytically. Nonlinear conjugate gradient and quasi-Newton methods, as local optimization methods, have faster convergence rates, but suffer from nonuniqueness; different starting points may result in different solutions. Hence, good prior information is needed for the local minimization algorithms to reach the global minimum. On the other hand, VFSR, which is based on a random sampling of the model space, has a slower convergence rate but guarantees convergence to the global minimum. VFSR is robust, independent from the starting point and only requires a physically meaningful range of the model parameters from which to sample.

Figure 7 shows the convergence rate of the objective function (5) for conjugate gradient and quasi-Newton methods for several runs from measured velocities at $40 \mathrm{MPa}$. The prior information for model parameters is taken randomly from a uniform distribution. Each run is started from a different random point and the minimization is continued until there is no further improvement in the model parameters. It is noticeable that wherever the starting point is far from the solution, the gradient methods are unlikely to converge to the global minimum. However, the convergence rate is very fast and it only takes a few iterations to reach the solution.

As an alternative approach, we have parameterized equation 2 in terms of Thomsen anisotropy parameter $\delta$ and anisotropy parameter $\eta$ (Alkhalifah and Tsvankin, 1995),

$$
\eta=\frac{\varepsilon-\delta}{1+2 \delta},
$$

by substituting for the Thomsen anisotropy parameter $\varepsilon$. This parameterization allows us to investigate how reliably $\eta$ can be

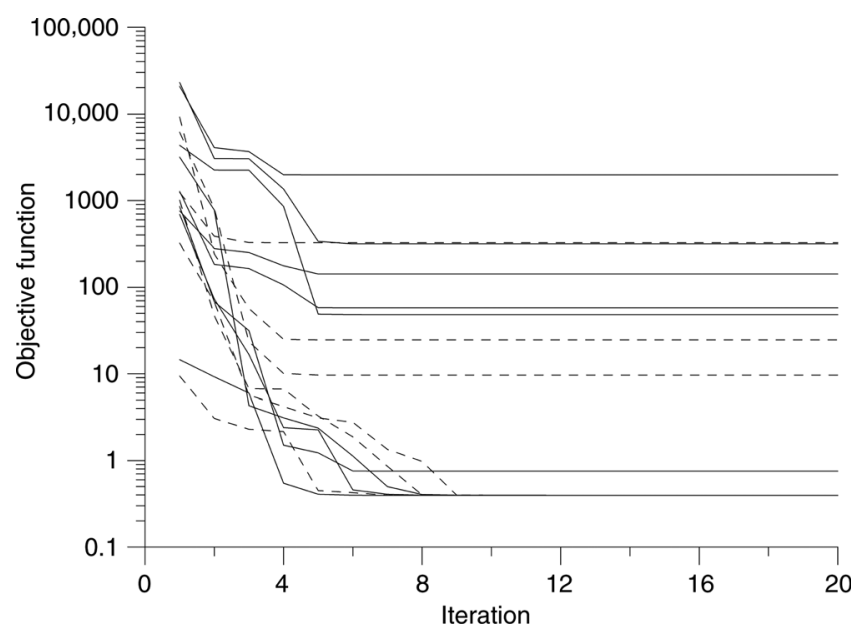

Figure 7. Convergence rate of the objective function from the minimization using the nonlinear conjugate gradient (dashed lines) and quasi-Newton (solid lines) algorithms. Only the lines that have the smallest value are to be considered to reach the global minimum. Because of nonuniqueness, the rest converged to local minima. 
estimated, as compared to computing it from $\varepsilon$ and $\delta$. We also are interested in seeing how $\eta$ influences the estimation of the other parameters, in particular $\delta$, and whether it improves the reliability of the inversion. The convergence patterns of the anisotropy parameters for both approaches, using the same data set, are shown in Figures 8-13.

In this example we continued the minimization up to 15,000 iterations; however, it is clear that the model parameters converged at very early iterations. In some cases, where trapped in local minima - as indicated in Figures $8-13$ by spikes, they could jump out quickly and back to the global minimum. It seems that rescaling the temperature and annealing time forces the algorithm to sample Thomsen anisotropy parameters mostly around the global minimum.

A quick inspection of the objective function of different iterations for both approaches shows that the anisotropy parameters

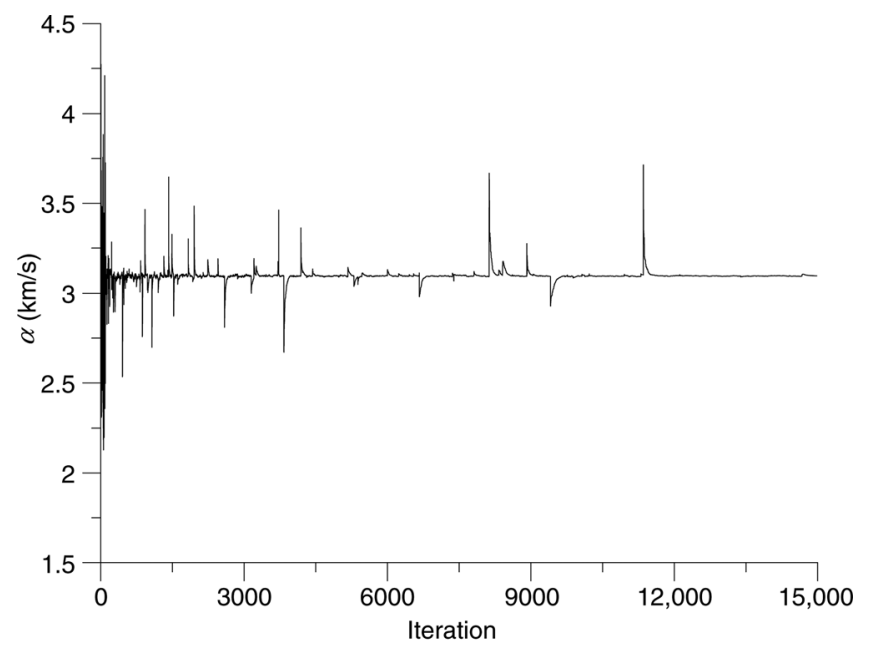

Figure 8. Convergence pattern of the Thomsen anisotropy parameter $\alpha$ from VFSR. The parameter $\alpha$ converged to the true solution at earlier iterations.

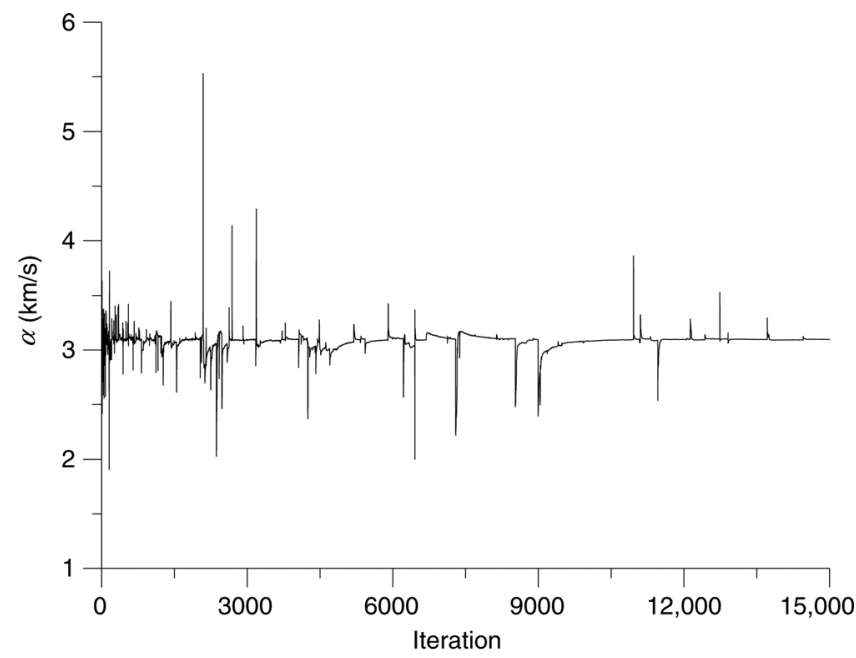

Figure 9. Convergence pattern of the Thomsen anisotropy parameter $\alpha$ from VFSR after parameterization. The parameter $\alpha$ converged to the true solution at earlier iterations. converged to exactly the same solution but in different iterations. In the first approach, where equation 2 is parameterized in terms of $\alpha, \varepsilon$, and $\delta$, the true solution is reached at iteration 6914, while in the second approach where $\alpha, \eta$, and $\delta$ are optimized, the true solution is reached at iteration 5318. The anisotropy parameter $\eta$, where computed from expression 6 , is the same exactly as the one estimated in the second approach.

Because of the stochastic nature of VFSR, every realization is different than the other; however all converge to the same solution. A quick comparison of the convergence pattern of the Thomsen anisotropy parameter $\delta$ from the two parameterization approaches, as illustrated in Figures 12 and 13, shows the superiority of the first approach. In the second approach, whenever $\delta$ is trapped in a local minimum, it takes a long time to return to global minimum. A few trials also showed the chaotic nature of the convergence pattern of anisotropy parameters in the second

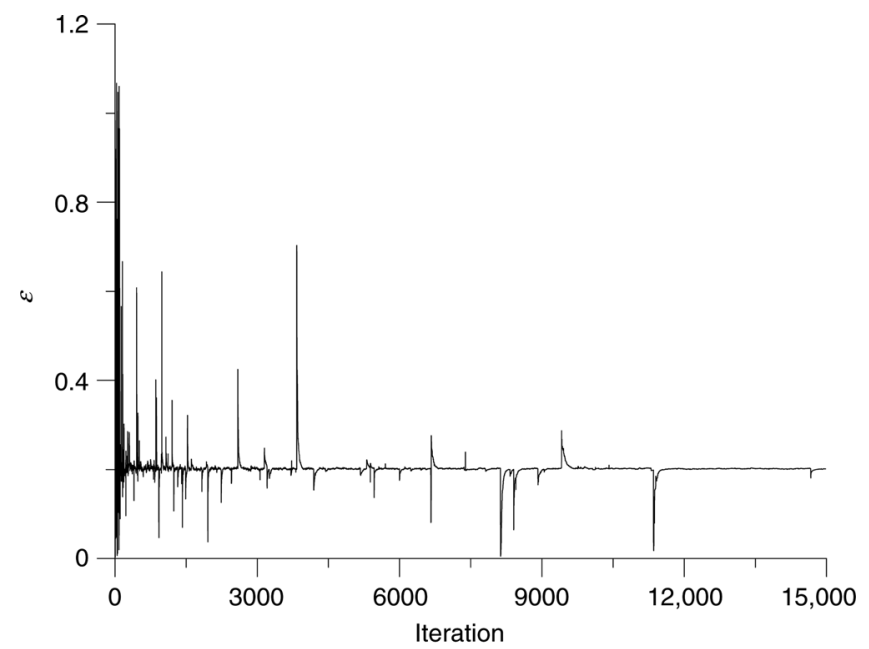

Figure 10. Convergence pattern of the Thomsen anisotropy parameter $\varepsilon$ from VFSR. The parameter $\varepsilon$ converged to the true solution at earlier iterations.

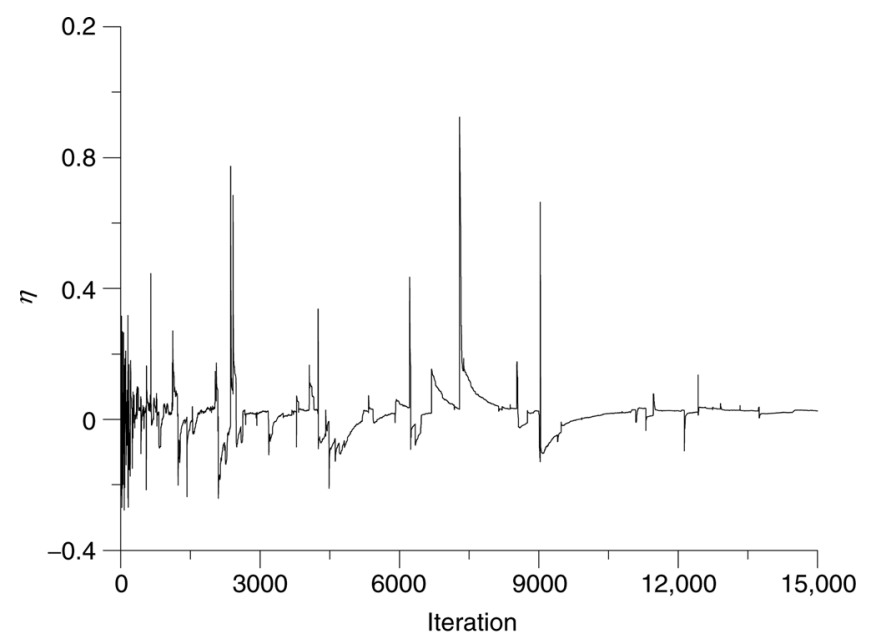

Figure 11. Convergence pattern of the anisotropy parameter $\eta$ from VFSR. The parameter $\eta$ takes more iterations to return to the true solution after getting trapped in a local minima. 
approach despite the convergence of all the model parameters to the true solution. This instability in convergence also makes it difficult to find a region close to the true solution to compute the uncertainty, as discussed later in the paper. The instability of the inversion in the second approach could be because of the division of two small parameters in the expression for $\eta$.

We note that the lower and upper bands of the model parameters for $\varepsilon$ and $\alpha$ could have been set closer, according to the velocity values estimated from the measurements along the symmetry axis/plane, but this reduction in the parameter space would not reduce the inversion time because of the stochastic nature of the sampling. A summary of the inversion process is illustrated by a flow chart in Figure 14.

\section{Pressure dependency of anisotropy parameters}

Figure 15 shows variation of the Thomsen anisotropy parameter $\alpha$ with changing confining pressure $P_{c}$. The velocity $\alpha$ increases exponentially as the pressure increases from the ambient pressure up to $100 \mathrm{MPa}$ and continues to increase linearly up to $400 \mathrm{MPa}$. Exponential behavior of the velocity dependence on stress is addressed by many authors (e.g., Eberhart-Phillips et al., 1989; Freund, 1992; Jones, 1995; Khaksar et al., 1999; Shapiro, 2003). We can use the formalism of Shapiro (2003) to interpret this in terms of the compliant and stiff porosities,

$$
V=A+K P_{e}-B e^{\left(-P_{e} D\right)},
$$

where $\mathrm{A}, \mathrm{K}, \mathrm{B}$, and $\mathrm{D}$ are fitting parameters, $P_{e}=P_{c}-P_{p}$ is the effective pressure, and $P_{p}$ is the pore pressure. Because the spherical sample was partially saturated, we might approximate the effective pressure, $P_{e} \approx P_{c}$. Given the applicability of Shapiro's formalism to the shales, the exponential increase in $\alpha$ is due mostly to the compliant porosity, where the microcracks and grain contacts close, while the linear part of the curve is more controlled by the stiff porosity. Figure 15 also shows that the exponential model fits very well with the estimated velocities along the symmetry axis with the parameters $A=3.243$, $K=0.00256, B=1.06, D=0.0403$.

Figures 16 and 17 show the changes of $\varepsilon$ and $\delta$ with pressure. A strong anisotropy can be seen at lower pressures and it decreases as the confining pressure increases. Up to $10 \mathrm{MPa}, \delta$ always is greater than $\varepsilon$; however this behavior is reversed for higher pressures. At $10 \mathrm{MPa}$, the spherical sample shows elliptical anisotropy, where $\delta=\varepsilon$. At higher pressures, in particular at $400 \mathrm{MPa}$, the shale sample still shows some degree of anisotropy, which could be caused by the intrinsic anisotropy of the clay minerals.

The pressure-dependence of the anisotropy parameter $\eta$ is shown in Figure 18. Up to $20 \mathrm{MPa}, \eta$ always is increasing. However as pressure increases $\eta$ behaves more pressure independent, and fluctuates around a very mildly dipping downtrend. The pressure dependence of the anisotropy parameters is summarized in Table 2.

\section{Uncertainty estimation}

To calculate the uncertainty of the estimation of the anisotropy parameters, we have used the approach given by Amand and Virieux (1997) with the last 2000 samples from VFSR to compute the covariance matrix. The square root of the diagonal elements of the covariance matrix (standard deviation) at each pressure is a statistical measurement of uncertainty for each estimated parameter.

Because in the first approach, the anisotropy parameter $\eta$ is not part of the minimization procedure, we approximate its uncertainty from the joint uncertainties associated with Thomsen anisotropy parameters $\varepsilon$ and $\delta$ by taking the partial differential of $\eta$ (expression 6) with respect to $\varepsilon$ and $\delta$,

$$
\Delta \eta \approx \frac{1}{1+2 \delta} \Delta \varepsilon-\frac{1+2 \eta}{1+2 \delta} \Delta \delta,
$$

where $\Delta \varepsilon \approx \sigma_{\varepsilon}$ and $\Delta \delta \approx \sigma_{\delta}$. The symbols $\sigma_{\varepsilon}$ and $\sigma_{\delta}$ are the uncertainties associated with the Thomsen anisotropy parameters $\varepsilon$ and $\delta$, respectively, computed from VFSR.

A fairly stable range of model parameters in the vicinity of the global minimum, in particular for $\delta$ and $\eta$, is needed to esti-

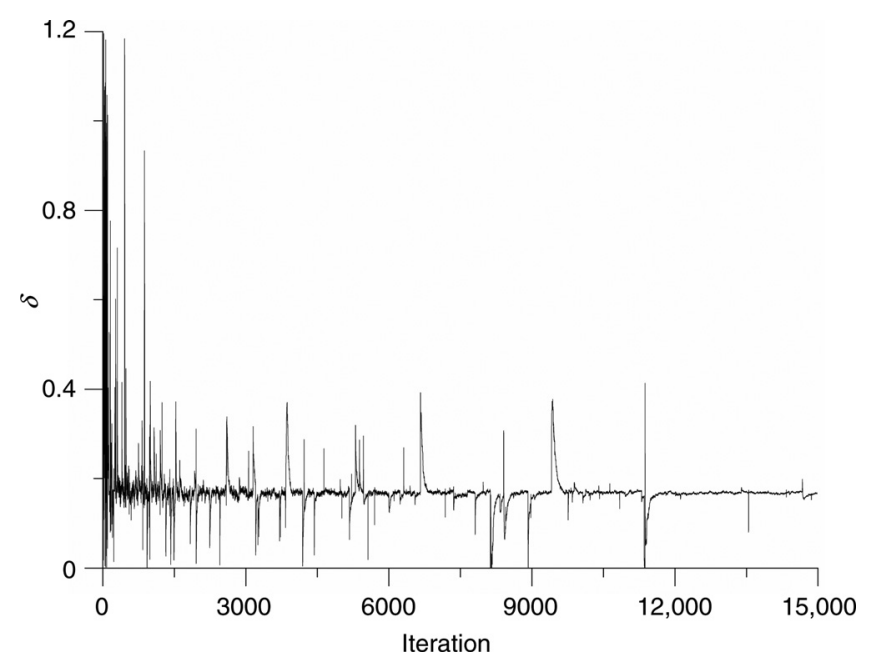

Figure 12. Convergence pattern of the Thomsen anisotropy parameter $\delta$ from VFSR. The parameter $\delta$ takes more iterations to converge to the true solution.

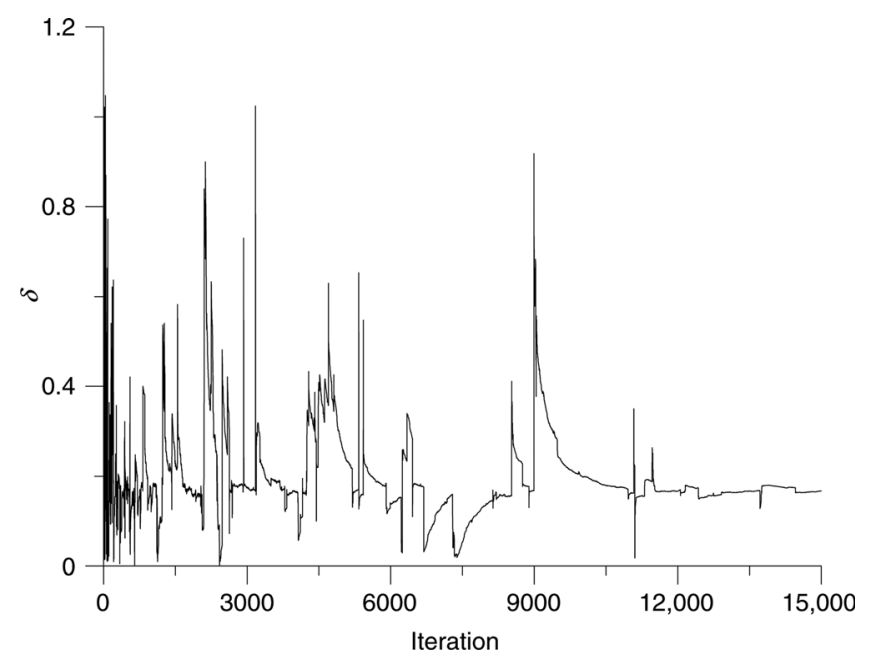

Figure 13. Convergence pattern of the Thomsen anisotropy parameter $\delta$ from VFSR after parameterization. The parameter $\delta$ takes more iterations to return to the true solution after getting trapped in a local minima. 
Figure 14. Flow chart showing the procedure for the estimation of the Thomsen anisotropy parameters.

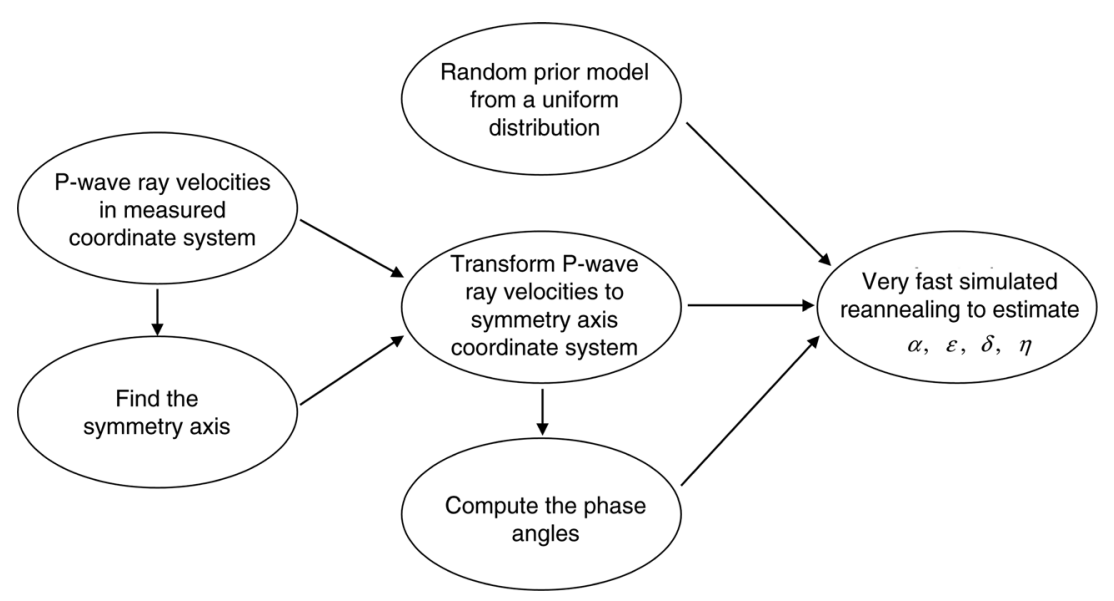

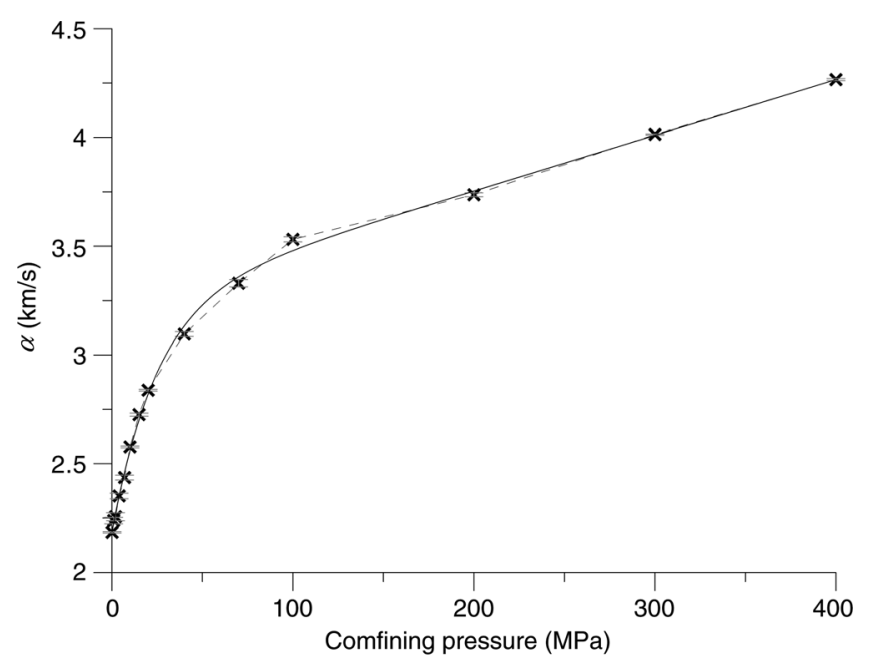

Figure 15. The Thomsen anisotropy parameter $\alpha$ and the uncertainties estimated at different confining pressures. Shapiro (2003) model (solid line) is fitted with $R^{2}=0.999, \quad \alpha=3.243$ $+0.00256 P_{c}-1.06 e^{\left(-0.0403 P_{e}\right)}$.

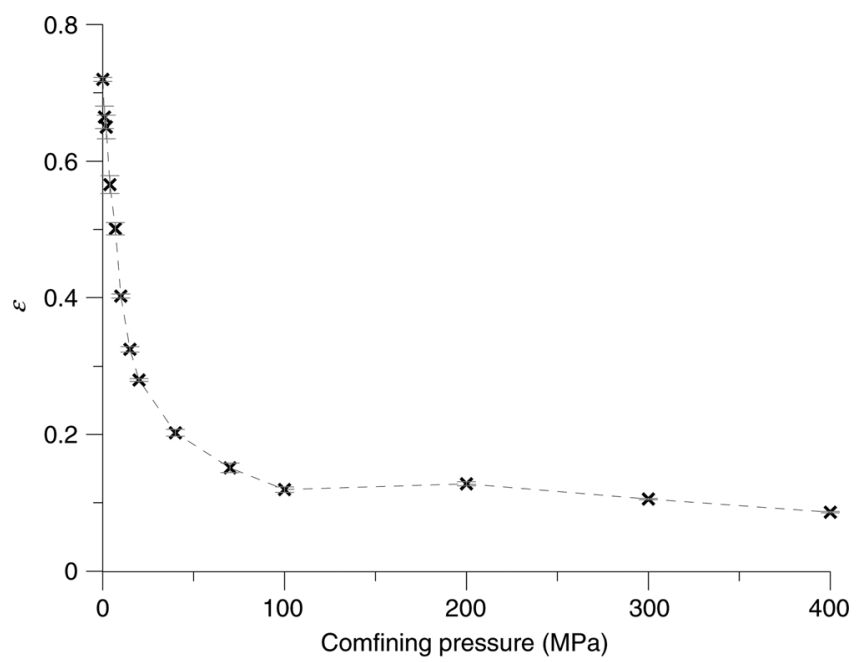

Figure 16. The Thomsen anisotropy parameter $\varepsilon$ and the uncertainties estimated at different confining pressures.

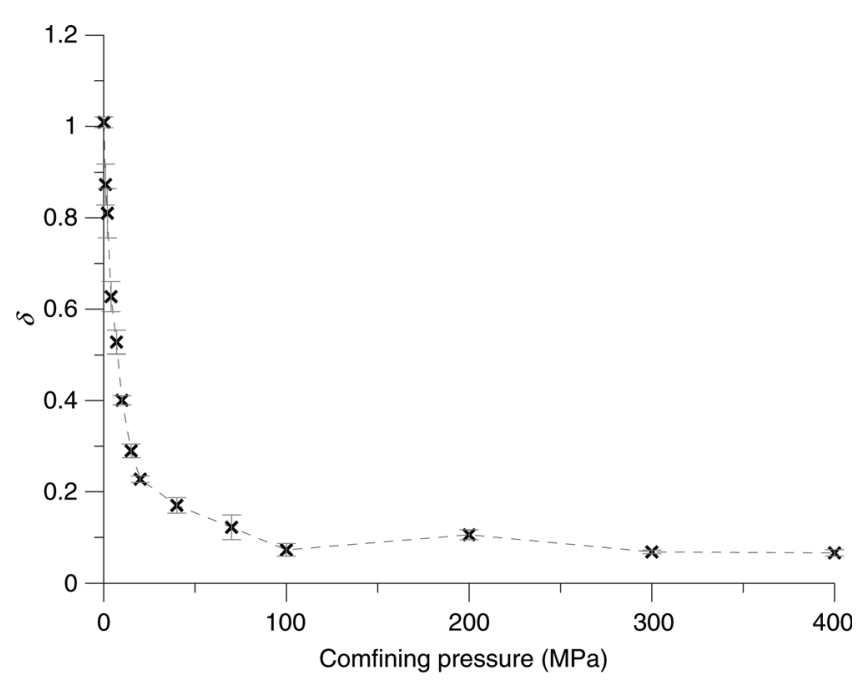

Figure 17. The Thomsen anisotropy parameter $\delta$ and the uncertainties estimated at different confining pressures.

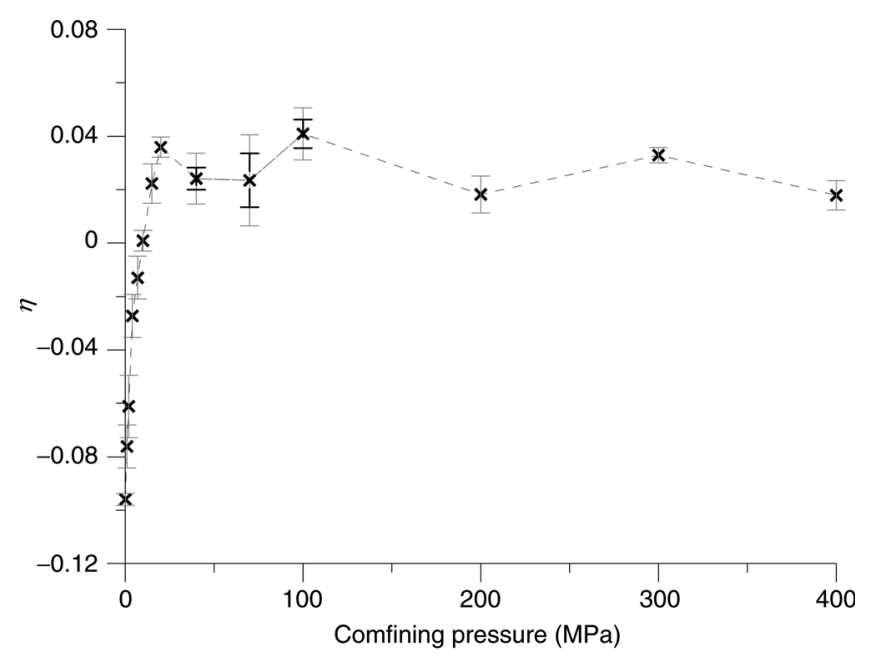

Figure 18. Anisotropy parameter $\eta$ and the uncertainties at different confining pressures. The thick bars are estimated from the VFSR, while the thin bars are computed from the uncertainty associated with $\varepsilon$ and $\delta$. 
Table 2. The Thomsen anisotropy parameters $\varepsilon, \delta, \alpha$ and their uncertainties at different pressures. ${ }^{4}$

\begin{tabular}{lccccccccccc}
$P_{c}(\mathrm{MPa})$ & $\varepsilon$ & $\delta$ & $\alpha$ & $\eta$ & $\sigma_{\varepsilon}$ & $\sigma_{\delta}$ & $\sigma_{\delta}^{*}$ & $\sigma_{\alpha}$ & $\sigma_{\alpha}^{*}$ & $\sigma_{\eta}$ & $\sigma_{\eta}^{*}$ \\
\hline 0.1 & 0.720 & 1.009 & 2.183 & -0.096 & 0.003 & 0.012 & - & 0.003 & - & 0.002 \\
1 & 0.664 & 0.873 & 2.238 & -0.076 & 0.016 & 0.045 & - & 0.015 & - & 0.008 & - \\
2 & 0.650 & 0.810 & 2.256 & -0.061 & 0.017 & 0.054 & - & 0.019 & - & 0.012 & - \\
4 & 0.566 & 0.627 & 2.351 & -0.027 & 0.013 & 0.033 & - & 0.013 & - & 0.008 & - \\
7 & 0.501 & 0.528 & 2.436 & -0.013 & 0.009 & 0.026 & - & 0.011 & - & 0.008 \\
10 & 0.402 & 0.401 & 2.577 & 0.001 & 0.003 & 0.010 & - & 0.004 & - & 0.004 \\
15 & 0.325 & 0.290 & 2.726 & 0.022 & 0.004 & 0.015 & - & 0.007 & - & 0.007 \\
20 & 0.280 & 0.228 & 2.838 & 0.036 & 0.002 & 0.007 & - & 0.004 & - & 0.004 \\
40 & 0.202 & 0.170 & 3.096 & 0.024 & 0.005 & 0.017 & 0.007 & 0.011 & 0.006 & 0.010 & - \\
70 & 0.151 & 0.122 & 3.329 & 0.023 & 0.007 & 0.027 & 0.014 & 0.017 & 0.010 & 0.017 & 0.010 \\
100 & 0.119 & 0.072 & 3.531 & 0.041 & 0.004 & 0.014 & 0.006 & 0.012 & 0.005 & 0.010 \\
200 & 0.128 & 0.106 & 3.737 & 0.018 & 0.003 & 0.011 & - & 0.009 & - & 0.007 \\
300 & 0.105 & 0.068 & 4.014 & 0.033 & 0.001 & 0.004 & - & 0.003 & - & 0.003 \\
400 & 0.086 & 0.066 & 4.265 & 0.018 & 0.001 & 0.007 & - & 0.005 & - & - \\
\hline
\end{tabular}

${ }^{4}$ The symbol $P_{c}$ is confining pressure, and $\sigma$ stands for standard deviation. The values of the $\alpha$ and its uncertainty are given in $\mathrm{km} / \mathrm{s}$. The uncertainty for the anisotropy parameter $\eta$ is computed from the uncertainties in $\varepsilon$ and $\delta$. The symbol $\sigma_{\eta}$ is the absolute value of the uncertainty in $\eta$. The expressions $\sigma_{\alpha}^{*}, \sigma_{\delta}^{*}$, and $\sigma_{\eta}^{*}$ are the uncertainties for the anisotropy parameters estimated from the parameterization of phase velocity in terms of $\alpha, \delta$, and $\eta$.

mate the uncertainty. This requires a large number of trials in the second approach, and thus we have investigated the effect of parameterization on the uncertainty of anisotropy parameters only at three different pressures. Wherever possible, the uncertainty of estimation of all the anisotropy parameters is lower than the uncertainty estimated from the first approach. The uncertainty is lower in particular for the anisotropy parameter $\eta$.

The uncertainties for the Thomsen anisotropy parameters $\alpha, \varepsilon$, $\delta$, and parameter $\eta$ are illustrated in Figures $15-18$ as the error bars for the whole range of the pressures. We also have plotted in Figure 18 uncertainty for the anisotropy parameter $\eta$, estimated from VFSR, at 40-100 MPa along with the uncertainties computed from expression 8. For a better comparison, the uncertainties also are shown in Table 2. The higher uncertainty of $\delta$ also is correlated well with Figure 6, in which the iso-surface is elongated mostly along the $\delta$ axis. This shows smaller sensitivity of the objective function (5) to the change in $\delta$, as compared to the other parameters in a simultaneous inversion.

\section{CONCLUSIONS}

We have estimated the Thomsen anisotropy parameters $\alpha, \varepsilon, \delta$, and anisotropy parameter $\eta$ for a spherical shale sample using the measured ultrasonic P-wave traveltimes in different azimuth and polar angles at confining pressures from 0.1 to $400 \mathrm{MPa}$. We assume that this spherical shale sample has transverse isotropy symmetry. The dependence of the residuals of the ray velocities on direction confirms the validity of this assumption (Bóna et al., 2010). We compute the phase angle from the measured ray velocity using numerical derivatives. This approach is extremely fast compared to ray tracing methods but may suffer from measurement errors. We have shown that this method works well for most of the pressure- dependent measurements. We estimate the symmetry axis assuming the invariance of ray velocities along the azimuth.

We have compared local and global minimization algorithms to estimate the Thomsen anisotropy parameters. The nonlinear conjugate gradient and quasi-Newton algorithms show similar fast performance. However, they suffer from nonuniqueness and their solutions often get trapped in local minima, unless the prior model is close to the solution. Very fast simulated reannealing has proven to overcome the nonuniqueness issue and converges to the global minimum. Local minimizations are very fast and converge to minima rapidly, unlike VFSR, which requires more iterations to reach the global minimum. We also have estimated the uncertainties associated with the estimation of the anisotropy parameters using VFSR.

We parameterized the phase velocity in terms of the anisotropy parameters $\delta$ and $\eta$ to investigate the stability of the inversion and the effect on the uncertainty. A few trials showed that the parameterization makes the objective function more complex with more local minima. Because of the stochastic nature of VFSR, whenever the sampling is in the vicinity of the global minimum for a long annealing time, it is possible to estimate the uncertainty fairly well and it often is lower than the uncertainty computed from expression 8. But this requires a large number of trials, which does not make this approach very attractive. Local gradient optimization algorithms often fail to reach the global minimum in parameterized approach, which also requires more trials.

A further investigation of the eigenvalues and the condition number of the matrix of the partial derivatives of the ray velocities with respect to the anisotropy parameters is required to see how ill-posed the parameterized inversion is.

We found that the Thomsen anisotropy parameter $\alpha$ depends exponentially on the confining pressure up to $100 \mathrm{MPa}$ and linearly from $100 \mathrm{MPa}$ to $400 \mathrm{MPa}$. According to Shapiro's formalism 
(Shapiro, 2003), these two types of behavior have been attributed to the closure of the compliant and stiff porosities, respectively. Shapiro's expression fits the estimated $\alpha$ with coefficient of determination $R^{2} \approx 1$, which supports the extension of Shapiro's formalism given by expression 7 to shales.

Thomsen anisotropy parameters $\varepsilon$ and $\delta$ decrease exponentially with pressure and possibly reach to the intrinsic anisotropy of the clay minerals at $400 \mathrm{MPa}$. Up to $10 \mathrm{MPa}, \delta$ always is greater than $\varepsilon$, but this trend reverses at higher pressures, which requires further investigations to explain. We found that despite hydrostatic pressure, symmetry axis direction changes noticeably with pressure, in particular at lower pressures. This change in the symmetry axis direction could be because of the closing of the microcracks with certain orientation; the characterization of such microcracks requires further studies.

\section{ACKNOWLEDGMENTS}

We thank BHP Billiton, in particular Guy Duncan, for partial financial support of this project. We especially thank Ivan Pšenčík, Leon Thomsen, and Boris Gurevich for their comments and recommendations during the 14th International Workshop on Seismic Anisotropy (14 IWSA), which motivated this work. We thank three anonymous reviewers and the associate editor for their comments and corrections that improved the quality of the paper. We thank Maxim Lebedev for providing the CT images.

\section{REFERENCES}

Alkhalifah, T., and I. Tsvankin, 1995, Velocity analysis for transversely isotropic media: Geophysics, 60, 1550-1566, doi:10.1190/1.1443888.

Amand, P., and J. Virieux, 1997, Nonlinear inversion of seismic reflection data by simulated annealing, in G. Chavent, and P. C. Sabatier, eds., Inverse problems of wave propagation and diffraction: Springer-Verlag, 323-333.

Arts, R. J., 1993, A study of general anisotropic elasticity in rocks by wave propagation - Theoretical and experimental aspects: Ph.D. thesis, Paris University.

Arts, R. J., P. N. J. Rasolofosaon, and B. E. Zinszner, 1991, Complete inversion of the anisotropic elastic tensor in rocks: Experiment versus theory: 61st Annual International Meeting, SEG, Expanded Abstracts, 1538-1541.

_ 1996, Experimental and theoretical tools for characterizing anisotropy due to mechanical defects in rocks under varying pore and confining pressures, in E. Fjaer, R. M. Holt, and J. S. Rathore, eds., Seismic anisotropy: SEG, $384-432$.

Berryman, J. G., 1979, Long-wave elastic anisotropy in transversely isotropic media: Geophysics, 44, 896-917, doi:10.1190/1.1440984.

Bóna, A., I. Bucataru, and M. A. Slawinski, 2007, Material symmetries versus wavefront symmetries: Quarterly Journal of Mechanics and Applied Mathematics, 60, 73-84, doi:10.1093/qjmam/hbl020.

Bóna, A., D. Nadri, and M. Brajanovski, 2010, Thomsen's parameters from p-wave measurements in a spherical sample: Geophysical Prospecting, doi:10.1111/j.1365-2478.2010.00917.x.

Červený, V., 1972, Seismic rays and ray intensities in inhomogeneous anisotropic media: Geophysical Journal of the Royal Astronomical Society, 29, 1-13.

Ciz, R., and S. A. Shapiro, 2009, Stress-dependent anisotropy in transversely isotropic rocks: Comparison between theory and laboratory experiment on shale: Geophysics, 74, no. 1, D7-D12, doi:10.1190/1.3008546.

Dellinger, J., D. Vasicek, and C. Sondergeld, 1998, Kelvin notation for stabilizing elastic-constant inversion: Revue de l'Institut Français du Pétrole, 53, 709-719.

Dewhurst, D. N., and A. F. Siggins, 2006, Impact of fabric, microcracks and stress field on shale anisotropy: Geophysical Journal International, 165, 135-148, doi:10.1111/j.1365-246X.2006.02834.x.

Eberhart-Phillips, D., D.-H. Han, and M. D. Zoback, 1989, Empirical relationships among seismic velocity, effective pressure, porosity, and clay content in sandstone: Geophysics, 54, 82-89, doi:10.1190/1.1442580.

Freund, D., 1992, Ultrasonic compressional and shear velocities in dry clastic rocks as a function of porosity, clay content, and confining pressure: Geophysical Journal International, 108, 125-135, doi:10.1111/ j.1365-246X.1992.tb00843.x.
Hornby, B. E., 1998, Experimental laboratory determination of the dynamic elastic properties of wet, drained shales: Journal of Geophysical Research, 103, 29945-29964, doi:10.1029/97JB02380.

Ingber, L., 1989, Very fast simulated re-annealing: Mathematical and Computer Modelling, 12, 967-973, doi:10.1016/0895-7177(89)90202-1.

Jech, J. Í., 1991, Computation of elastic parameters of anisotropic medium from travel times of quasi-compressional waves: Physics of the Earth and Planetary Interiors, 66, 153-159, doi:10.1016/00319201(91)90074-R.

Jones, L. E. A., and H. F. Wang, 1981, Ultrasonic velocities in Cretaceous shales from the Williston basin: Geophysics, 46, 288-297, doi:10.1190/ 1.1441199 .

Jones, S. M., 1995, Velocities and quality factors of sedimentary rocks at low and high effective pressures: Geophysical Journal International, 123, 774-780, doi:10.1111/j.1365-246X.1995.tb06889.x.

Khaksar, A., C. M. Griffiths, and C. McCann, 1999, Compressional- and shear-wave velocities as a function of confining stress in dry sandstone: Geophysical Prospecting, 47, 487-508, doi:10.1046/j.1365-2478.1999. 00146.x.

Lokajíček, T., and K. Klíma, 2006, A first arrival identification system of acoustic emission (AE) signals by means of a high-order statistics approach: Measurement Science \& Technology, 17, 2461-2466, doi:10.1088/0957-0233/17/9/013.

Lucet, N., 1989, Vitesse et des ondes elastiques soniques et ultrasoniques dans les roches sous pression de confinement: Ph.D. thesis, University of Pierre and Marie Curie.

Lucet, N., and B. E. Zinszner, 1992, Effects of heterogeneities and anisotropy on sonic and ultrasonic attenuation in rocks: Geophysics, 57, 1018-1026, doi:10.1190/1.1443313.

Nocedal, J., and S. J. Wright, 1999, Numerical optimization: Springer-Verlag.

Prioul, R., A. Bakulin, and V. Bakulin, 2004, Nonlinear rock physics model for estimation of 3D subsurface stress in anisotropic formations: Theory and laboratory verification: Geophysics, 69, 415-425, doi:10.1190/1.1707061.

Pros, Z., and V. Babuška, 1967, A method for investigating the elastic anisotropy on spherical rock samples: Zeitschrift für Geophysik, 33, 289-291.

, 1968, An apparatus for investigating the elastic anisotropy on spherical rock samples: Studia Geophysica et Geodaetica, 12, 192-198, doi:10.1007/BF02587847.

Pros, Z., T. Lokajíček, and K. Klíma, 1998, Laboratory approach to the study of elastic anisotropy on rock samples: Pure and Applied Geophysics, 151, 619-629, doi:10.1007/s000240050133.

Pros, Z., and Z. Podroužková, 1974, Apparatus for investigating the elastic anisotropy on spherical rock samples at high pressure: Veröffentlichungen des Zentralinstituts für Physik der Erde, Potsdam, 22, 42-47.

Rasolofosaon, P. N. J., and B. E. Zinszner, 2002, Comparison between permeability anisotropy and elasticity anisotropy of reservoir rocks: Geophysics, 67, 230-240, doi:10.1190/1.1451647.

Sarkar, D., A. Bakulin, and R. L. Kranz, 2003, Anisotropic inversion of seismic data for stressed media: Theory and a physical modeling study on Berea Sandstone: Geophysics, 68, 690-704, doi:10.1190/1.1567240.

Shapiro, S. A., 2003, Elastic piezosensitivity of porous and fractured rocks: Geophysics, $\mathbf{6 8}, 482-486$, doi:10.1190/1.1567216.

Tarantola, A., 2005, Inverse problem theory and methods for model parameter estimation: SIAM.

Thill, R. E., T. R. Bur, and R. C. Steckley, 1973, Velocity anisotropy in dry and saturated rock spheres and its relation to rock fabric: International Journal of Rock Mechanics and Mining Sciences \& Geomechanics Abstracts, 10, 535-557, doi:10.1016/0148-9062(73)90004-1.

Thill, R. E., R. J. Willard, and T. R. Bur, 1969, Correlation of longitudinal velocity variation with rock fabric: Journal of Geophysical Research, 74, 4897-4909, doi:10.1029/JB074i020p04897.

Thomsen, L., 1986, Weak elastic anisotropy: Geophysics, 51, 1954-1966, doi:10.1190/1.1442051

Tsvankin, I., 2005, Seismic signatures and analysis of reflection data in anisotropic media: Elsevier.

Ursin, B., and K. Hokstad, 2003, Geometrical spreading in a layered transversely isotropic medium with vertical symmetry axis: Geophysics, $\mathbf{6 8}$, 2082-2091, doi:10.1190/1.1635062.

Vestrum, R. W., 1994, Group- and phase-velocity inversions for the general anisotropic stiffness tensor: M.S. thesis, University of Calgary.

Vestrum, R., R. J. Brown, and D. T. Easly, 1996, From group or phase velocities to the general anisotropic stiffness tensor, in E. Fjaer, R. M. Holt, and J. S. Rathore, eds., Seismic anisotropy: SEG, 101-140.

Vickers, B. L., and R. E. Thill, 1969, A new technique for preparing rock spheres: Journal of Physics E: Scientific Instruments, 2, 901-902, doi:10.1088/0022-3735/2/10/422.

Wang, Z., 2002a, Seismic anisotropy in sedimentary rocks, part 1: A single-plug laboratory method: Geophysics, 67, 1415-1422, doi:10.1190/ 1.1512787

, 2002b, Seismic anisotropy in sedimentary rocks, part 2: Laboratory data: Geophysics, 67, 1423-1440, doi:10.1190/1.1512743. 